Review

\title{
Moderate the MAOA-L Allele Expression with CRISPR/Cas9 System
}

\author{
Martin L. Nelwan \\ Department of Animal Science - Other \\ Nelwan Institution for Human Resource Development \\ Jl. A. Yani No. 24 \\ Palu, Sulawesi Tengah, Indonesia \\ Email: mlnelwan2@gmail.com
}

\begin{abstract}
Antisocial behavior is a behavior disorder inherited according to the inheritance of X-linked chromosome. This disorder derives from mutations in the MAOA gene. One of the mutations is the $M A O A-L$ allele and result in MAOA-L activity. The MAOA-L allele activity can cause antisocial behavior in both healthy and unhealthy people. Antisocial behavior from healthy males can originate from maltreatment during childhood. Currently, MAOA inhibitor can reverse antisocial behavior to normal behavior in animal models. However, this disorder cannot be treated permanently; to treat it permanently in the future, technologies such as CRISPR/Cas9, iPSCs and ssODN are required. These technologies have succeeded to correct erroneous segments in the F8 gene and F9 gene. Both genes occupy the $\mathrm{X}$ chromosome. The $M A O A$ gene also occupies the $\mathrm{X}$ chromosome. It is reasonable to state that CRISPR/Cas9 and iPSCs technique for instance can be beneficial tools to edit the MAOA gene to treat antisocial behavior. CRISPR/Cas9 can be used in combination with iPSCs or ssODN for instance. This combination can greatly help the permanent healing of antisocial behavioral disorders.
\end{abstract}

Keywords: advanced therapy; aggressive; antisocial; behavior; MAOA.

\section{Introduction}

Antisocial behavior is a hereditary disorder inherited through an X-linked recessive inheritance pattern. The MAOA gene has correlation with this antisocial behavior [1, 2]. Mutations in this gene result in low MAOA (MAOA-L) expression [3]. These mutations can create the MAOA-L allele. The MAOA-L expression affects males nearly entirely and can result in behavior problems such as aggressive and violent eruptions $[2,3]$. Antisocial behavior can exist in each family. When parents are carrier female and normal male, $1 / 2$ female children are carriers and $1 / 2$ normal female. Male children are $1 / 2$ normal and $1 / 2$ antisocial behaviors. When parents are normal female and antisocial behavior male, all female children are carrying and all male children are antisocial behaviors (Figure 1). The $M A O A-L$ allele is outstandingly general and happens in about $40 \%$ [4] or $41 \%$ of the Caucasian people [5]. These people have peaceable behavior and have never committed a crime. A study has detected that at least males with this variant had neurobiological framework factors. These factors incline them to violent behavior [3] or antisocial behavior. Maltreatment in children with $M A O A-L$ allele can cause antisocial behavior.

In animal models, the MAOA inhibitor can reverse the antisocial behavior, suggesting that the $M A O A-L$ allele expression can be moderated permanently. To reverse antisocial behavior permanently, gene-editing techniques can be used. One of the gene-editing techniques is clustered regularly interspaced short palindromic repeats (CRISPR/Cas9) system. Others are meganucleases (MNs), zinc finger nucleases (ZFNs) and transcription activator-like effectors nucleases (TALENs) [6]. CRISPR/Cas9 
system gets the benefit of RNA-guided Cas9 nuclease to produce aimed double-stranded DNA breaks. In addition, the gene-editing technique in combination with induced pluripotent stem cells (iPSCs) or single-stranded oligonucleotides (ssODN) is useful to fix erroneous segment in genes such as $M A O A$ gene and F8 gene [6,7]. For example the CRISPR/Cas9 system in combination with iPSCs technique has shown its benefit to slow down hemophilia A. Hemophilia A is inherited according to the X-linked hereditary. Therefore, it is reasonable to state that the iPSCs in combination with CHRISPR/Cas9 system may be useful to moderate the antisocial behavior in humans, for instance.

P:

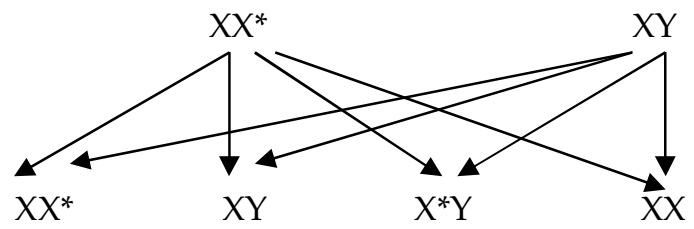

F2:

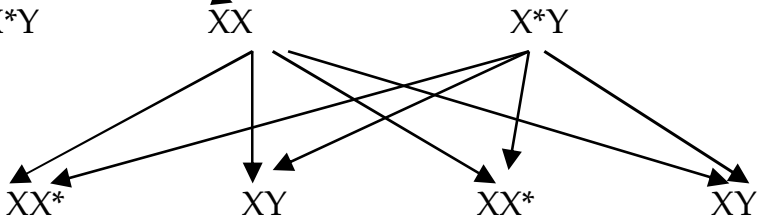

Figure 1. In $\mathrm{F}_{1}, 50 \%$ female is carrier and $50 \%$ is normal. $50 \%$ male is normal and $50 \%$ are affected. In $\mathrm{F}_{2}$, all female is carrier and all male is normal.

In this article, the author describes progress in the study of antisocial behavior. The author focuses on the biological aspects and gene therapy. The biological aspects include the MAOA gene, mutations in the $M A O A$ gene and antisocial behavior and treatment with gene therapy. Gene therapy includes CRISPR/Cas9 system in combination with iPSCs or HR-based method.

\section{Genes in Antisocial Behavior}

Gene is fundamental bodily and functional unit of heredity. Genes consist of DNAs and supply instructions to build protein molecules. Changes can occur in a gene and can cause protein destruction. A gene change is a stable mutation in the DNA. A condition derives from changes in at least one gene stated as a hereditary disease [8]. For example, hereditary diseases can include such as hemophilia $\mathrm{A}$ and antisocial behavior. Antisocial behavior derived from the mutation of the $M A O A$ gene into the $M A O A-L$ allele.

\subsection{The MAOA Gene}

"Monoamine oxiadase $\mathrm{A}^{\prime}$ is the formal name of the $M A O A$ gene. $M A O A$ is the gene formal symbol. Other names of these genes include $B R N R S$ and $M A O-A$ [3] The MAOA gene spans at least $60 \mathrm{~kb}$. This gene consists of 15 exons. The $M A O A$ gene displays the same exon-intron organization. Exon 12 encodes for the covalent FAD-binding site. This exon is the most conserved exon [9-10]. The MAOA gene occupies the $\mathrm{p}$ arm of the $\mathrm{X}$ chromosome at position 11.3; Xp11.3 [9]; Figure 2. This gene includes base pairs $43,654,907$ to $43,746,824$ on the $X$ chromosome $[3,11]$.

The $M A O A$ gene is one of two neighboring gene families. The other gene is $M A O B$. The $M A O A$ and $M A O B$ derived from duplication of the $M A O$ gene [10]. The external mitochondrial membrane expresses these two genes [9-10]. Chen et al stated that these two genes oxidize neurotransmitters and dietary amines [10]. The regulation of neurotransmitters activity is vital in sustaining standard mental conditions $[3,10]$. Chen et al localized the $M A O A$ and $M A O B$ genes within a region of about $240 \mathrm{~kb}$. The 
$M A O A$ gene encodes mitochondrial enzymes and catalyzes the oxidative deamination of amines [9-10]. These include such as dopamine, norepinephrine, and serotonin as substrates [3, 10-13]. The MAOB gene prefers phenylethylamine as substrates $[10,13]$.

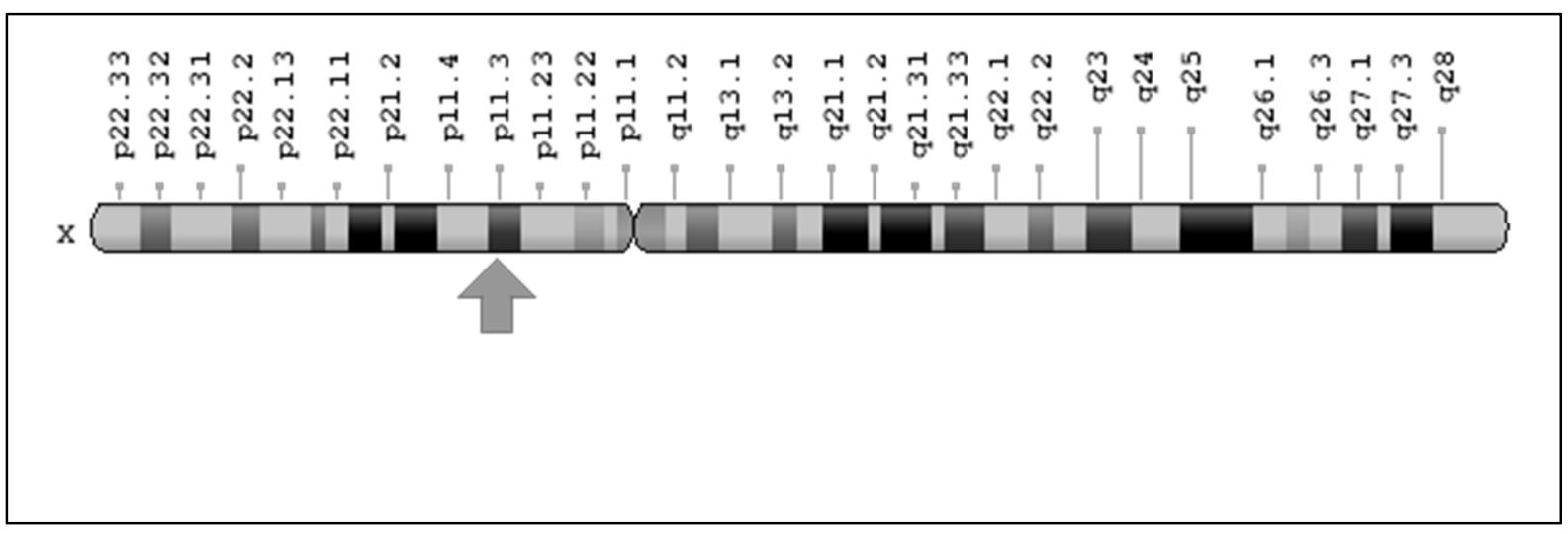

Figure 2; The $M A O A$ gene; The $M A O A$ gene location on $\mathrm{X}$ chromosome at position 11.3 (from reference [3]).

The MAOA gene has significant roles in the metabolism of neuroactive and vasoactive amines. This metabolism occurs in the central nervous system and peripheral tissues $[3,8]$. Ou et al., established that serum hunger-made apoptosis in culture neuronal cell line enhanced demonstration of MAOA. In addition, this serum enhanced demonstration of p38 kinase and caspase-3. This apoptosis diminished bd2 and R1. MAOA and R1 were upstream of caspase-3. Both of them were downstream of p38 kinase and BCL2 in the apoptotic signaling pathway. Moreover, Ou et al., stated that serum starvation of cortical brain cells from Maoa-deficient mice resulted in reduced apoptosis contrasted with wild-type (WT) mice. $M A O A$ and R1 were involved in the MYC-made proliferative signaling pathway in the attendance of serum. Both of them function upstream of cyclin D1 and E2F1 in the cell proliferation pathway. The $M A O A$ inhibitor could avoid apoptosis. [10,14].

\subsection{Mutations in the MAOA Gene for the Antisocial Behavior}

Antisocial behavior can happen in healthy people $[2,15]$. Antisocial behavior is cross-transmitted with other dyscontrol disorders. This behavior has several well-defined biological correlates. These include injured frontal lobe function, leading to reduced ability to control behavior. It diminishes levels of the serotonin in cerebro-spinal fluid. Furthermore, it exhibits moderate heritability. Aggression is an important manifestation of antisocial behavior. [15]. Caspi et al studied male children from birth to adulthood subjected to maltreatment. The authors stated that maltreated children with a genotype conferring the $M A O A-H$ allele expression were less likely to develop antisocial behavior. It shows that children with the $M A O A-L$ allele expression correspond to antisocial behavior [10, 16]. This means that environment has important role developing antisocial behavior in humans.

In 1981, Pintar et al allocated the MAOA gene on the human X chromosome [5, 10, 17]. Later, the $M A O A-L$ allele activity and antisocial behavior in male mice with an $\mathrm{X}$ chromosome deletion were linked. Cases et al reported that deletion in the $M A O A$ gene in mice revealed an increase of norepinephrine, serotonin and dopamine. Moreover, this mutation raised aggression in male mice $[10,17,18]$. Reti et al introduced that Caucasians with $M A O A-L$ allele had antisocial behavior around $41 \%$ [5]. It supports to a link between $M A O A-L$ allele and antisocial behavior. 
McDermott et al conducted a behavioral study in humans to link behavior and environment influence. In this study, the authors paid male subjects to penalize those they considered had stolen money from them. The authors adjusted the amount of money lost from them to their enemies. McDermott et al reported that people with MAOA-L had violent behavior penalize their enemies. The connection was critical when the quantity of money was higher, suggesting an environmental interaction. It shows that heredity can play a role in the behavior and daily decisions taken [10,19].

Ziemans et al stated that a single-nucleotide polymorphism (rs6609257) considerably influenced a brain network activity. This network includes frontal, parietal and occipital areas. The authors indicated that the rs6609257 occupies $\sim 6.6 \mathrm{~kb}$ downstream of the MAOA gene on the human $\mathrm{X}$ chromosome. Improved activity in this network had correlation with visuospatial working memory (VSWM) capacity in the order predicted externalizing symptoms. The authors showed that a higher working memory capacity had not associated with fewer externalizing symptoms. However, these externalizing symptoms associated with aggressive/oppositional behavior. In this study, the authors proposed a mediating function or working memory brain activity in connecting the $M A O A$ gene to aggressive behavior [20]. Furthermore, Marquez et al showed that male rats, which surrendered to pressure-induced experiences through peripubertal, show aggressive behavior at maturity. The authors indicated that treatment with an MAOA inhibitor reversed the peripuberty pressure-induced antisocial behaviors. Marquez et al showed that biological factors, which are activated through maltreatment, are the cause of antisocial behavior [21]. It suggests that education not to do violent behavior is very important. It can help to reduce "antisocial behavior" among people.

Maltreatment in childhood ( $\mathrm{G} \times \mathrm{E})$ can result in emotional and antisocial behavioral problems in youth. These people have low variability on the variable number tandem repeats (VNTR) polymorphism of the MAOA gene [22-23, 25-26]. The VNTR polymorphism in human consists of 30 base pairs in length. These include $2 \mathrm{R}, 3 \mathrm{R}, 3.5 \mathrm{R}, 4 \mathrm{R}$ and $5 \mathrm{R}$ copies of the repeat series. The polymorphism demonstrated influence on transcriptional activity of the $M A O A$ gene promoter. The $3.5 \mathrm{R}$ and $4 \mathrm{R}$ repeats are transcribed more effectively than those with $2 R$ and $3 R$ copies $[2,10,23,25-26]$. Males with a $2 R$ variant have a level of serious criminal behavior and violent behavior. Effects for females are alike, but weaker [10, 25]. The effect $5 \mathrm{R}$ is unclear [3]. The $2 \mathrm{R}$ promoter displays many inferior levels of promoter activity than the other promoters.

Behavior disorder due to abuse has contradictorily established a connection between the MAOA$L$ allele and antisocial behavior. The $M A O A-L$ allele activity raises the risk of behavior disorder and antisocial behavior traits. This happens to young people who experience maltreatment during childhood [22, 26-29]. In addition, non-linear interactions between the $M A O A$ gene and violence have been found [2, 27]. It suggests that people with the $M A O A-L$ allele can be hypocrite once a certain violent level are detected.

Three mutations occur in the MAOA-L allele so far. These include nonsense (Brunner syndrome), missense (autism), and a deletion (Norrie disease). Brunner syndrome and autism correspond to aggressive behavior. Brunner syndrome has antisocial behavior and autism has auto-aggressive behavior, while Norrie disease corresponds to autistic-like behavior. Brunner syndrome, autism and Norrie disease belong to intellectual disability (ID). Brunner syndrome shows stress-induced aggressive and violent behavior in addition to borderline ID [30]. This shows that antisocial behavior can include borderline behavior. Furthermore, antisocial behavior can exist both in healthy $[2,4]$ and in unhealthy people [30].

Transgenic mice for antisocial behavior researches can be obtained. Use of mice will be helpful to conduct research for treating disorders inherited through $X$-linked recessive configurations. These disorders can include such as hemophilia B and antisocial behavior. For example, to diminish antisocial behavior, advanced therapy such as CRISPR/Cas9 system in combination with iPSCs technology can be used. This combination can be helpful to treat diseases inherited through X-linked pattern [6-7]. 


\section{The Gene-Editing Technique}

Currently, four methods for targeted integration of transgenic are available. These include NHEJ, MMEJ, HR, and HMEJ (Table 1). The NHEJ-based method presented random directions in integration and various types of indels at the junctions. NHEJ is active in the entire cell cycle [35-36]. The MMEJbased method displayed low efficiency in cultured cells. MMEJ is active in the early S/G1 phase [35, 37]. The HR-mediated method allows correct insertion of large fragments. This method is commonly inefficient in animal embryos and tissues in vivo. HR is active during the late S/G2 phase only. Finally, the HMEJ-based method achieved transgenic integration in mouse and monkey embryos, as well as in hepatocytes and neurons in vivo with high efficiency. HMEJ is active in the early S/G1 phase. All of the methods can be useful for generating animal models and for targeted gene therapies [35].

Table 1. Methods for targeted integration transgenic

\begin{tabular}{cccc}
\hline Protocol & Advantage/disadvantage & Cell cycle & References \\
\hline HR & Inefficiency & S/G2 & {$[35]$} \\
MMEJ & High efficiency & Early S/G1 & {$[35]$} \\
NEHJ & Random directions & entire phase & {$[35,36]$} \\
HMEJ & Low efficiency & early S/G1 & {$[35,37]$} \\
\hline
\end{tabular}

\subsection{Gene-editing in X-inked Disorders}

The gene-editing method uses creator of nucleases to edit incorrect gene. This method also uses the cellular repair technique to exactly alter incorrect string. For example, to identify the selected genomic location and its transfected into the cell, an artificial chain-precise is designed. It produces double-strand breaks (DSBs) at the location [31].' The non-homologous end joining (NHEJ) [31, 32, 33, 34], microhomology-mediated end joining (MMEJ), homologous recombination (HR) [34] and homologymediated end joining (HMEJ) can repair DSBs. Nucleases such as TALENs and CRISPR/Cas9 systems can induce DSBs in a targeted genomic locus [35].

The $F 8$ gene and $F 9$ gene are genes that occupy the $\mathrm{X}$ chromosome. It is the same as the MAOA-L allele that also occupies the $\mathrm{X}$ chromosome in the chromosome map. Currently, with the development of CRISPR/Cas9 system, mutations in genes F8 and F9 can be corrected. For example, the CRISPR/Cas9 in combination with iPSCs or ssODN can correct erroneous segments in these genes in animal models. CRISPR/Cas9 in combination with iPSCs is gene-editing technique for hemophilia A. Park et al edited mutations in the F8 gene with this combination. These authors practically rescued the Factor VIII deficiency in a hemophilia mouse model [38]. CRISPR/Cas9 in combination with ssODN is gene-editing technique for hemophilia B. Guan et al generate mutated mouse strains for hemophilia B, and then cured these strains in vivo by hydrodynamic tail injection of a plasmid. The plasmid encodes Cas9 and the sgRNA in combination with ssODN containing the edited string [39]. It seems that these techniques can be potential tools to treat antisocial behavior.

\subsection{Gene-editing for Antisocial Behavior}

CRISPR/Cas9 system, iPSCs technique, and MHEJ-based method for instance can be useful tools to treat antisocial behavior in either healthy or unhealthy people. CRISPR/Cas9 system can correct the incorrect gene in iPSCs. CRISPR/Cas9 system cleaves the chromosome DNA, and then constructs DSBs. HMEJ will correct the nick. It will correct segments or remove the 30-bp copy in the string. This gene- 
corrected is differentiated into suitable somatic cells. Furthermore, patients can obtain the corrected gene by giving it to patients.

To alter the $M A O A-L$ allele needs gene-editing technique. In animal models, this technique is a useful tool to treat an X-linked recessive disorder such as hemophilia $\mathrm{A}$ and hemophilia B. The CRISPR/Cas9 system in combination with such as the iPSCs technique or HMEJ-based method is useful to fight antisocial behavior.

\section{Conclusions}

Antisocial behavior is a violent behavior inherited according to the inheritance of sex-linked recessive allele. A violent behavior derives from mutations in the $M A O A$ gene. These mutations can result in, such as autism, Brunner syndrome, and antisocial behavior. The $M A O A-L$ allele expression corresponds to antisocial behavior. Environmental factors such as maltreatment can cause antisocial behavior in male children. To treat permanently this behavior, it is impossible at present. In the future, to treat this behavior, a gene-editing tool such as TALENs or CRISPR/Cas9 systems can be used. For example, CRISPR/Cas9 system can correct erroneous segments in the sex-linked disorders. CRISPR/Cas9 system can be used in combination with iPSCs technique or HMEJ-based method for instance. For example, CRISPR/Cas9 in combination with iPSCs has corrected erroneous segments in the F8 gene in the animal models. These findings give hope to treat antisocial behavior with the CRISPPR/Cas9 system in combination with iPSCs technique for instance. It can alter the $M A O A-L$ allele to normal allele to result in normal behavior. This combination is a promising tool to treat permanently the antisocial behavior in both healthy and unhealthy people.

Acknowledgement: The author has role in designing of the study and writing of the manuscript.

Author Contributions: MLN designed the study, performed the literature searches, wrote the first draft of the manuscript and involved in revising the manuscript critically for significant intellectual need. The author read and approved the final manuscript.

Conflict of Interests: The author declares no conflict of interest.

\section{References}

1. Xu, M.K.; Gaysina, D.; Tsonaka, R.; Morin, A.J.S.; Croudace, T.J.; Barnett, J.H.; et al. Monoamine Oxidase A $(M A O A)$ Gene and Personality Traits from Late Adolescence through Early Adulthood: A Latent Variable Investigation. Front. Psychol. 2017; 8: 1736, doi:10.3389/psyg.2017.01736.

2. Kolla, N.J.; Meyer, J.; Sanches, M.; Charbonneau, J. Monoamine Oxidase-A Genetic Variants and Childhood Abuse Predict Impulsiveness in Borderline Personality Disorder. Clinical Psychopharmacology and Neuroscience 2017; 15(4): 343-351, doi:10.9758/cpn.2017.15.4.3433.

3. Genetics Home Reference (2018): MAOA, https://ghr.nlm.nih.gov/gene/MAOA.

4. Hunter, P. The Psycho gene. EMBO reports 2010, 11 9: 667.

5. Reti, E.M.; Xu, J.Z.; Yanofski, J.; McKibben, J.; Uhart, M.; Cheng Y-J, et al. MAO regulates antisocial personality in Caucasians with no history physical abuse. Compr. Psychiatry 2011; 52(2): 188-194.

6. Nelwan, M.L. Hemophilia A and Induced Pluripotent Stem Cells. Journal of Advances in Biology $\mathcal{E}$ Biotechnology 2017; 14(3): 1-11, doi:10.9734/JABB/2017/35111.

7. Nelwan, M.L. Friedreich Ataxia: Treatment with Genetic Approach. Journal of Advances in Biology $\mathcal{E}$ Biotechnology 2017; 14(4): 1-12,doi:10.9734/JABB/2017/36113. 
8. Nelwan, M.L. Treat Oculocutaneous Albinism with Gene Therapy. Journal of Advances in Biology $\mathcal{E}$ Biotechnology 2017; 16(3): 1-12. DOI:10.9734/ JABB/2017/38504.

9. Grimsby, J.; Chen, K.; Wang, L-J.; Lan, N.C.; Shih, J.C. Human Monoamine Oxidase A and B genes exhibit identical exon-intron organization. Proc. Nat. Acad. Sci. 1991; 88: 3637-3641.

10. Online Mendelian Inheritance in Man (2017): Monoamine Oxidase A; MAOA, http://omim.org/entry/309850.

11. NCBI Gene, https://www.ncbi.nlm.nih.gov/gene/4128.

12. Chen, K.; Holschneider, D.P.; Wu, W.; Rebrin, I.; Shih, J.C. A spontaneous point mutation produces monoamine oxidase A/B knock-out mice with greatly elevated monoamines and anxiety-like behavior. J. Biol. Chem. 2004; 279: 39645-39652, doi:10.1074/jbc.M405550200.

13. UniProt, http://www.uniprot.org/uniprot/P21397.

14. Ou, X-M.; Chen, K.; Shih. J.C. Monoamine axidase A and repressor R1 are involved in appropriate signaling pathway. Proc. Nac. Acad. Sci. 2006; 103 (29): 10923-10928, doi:10.1073/pnas.0601515103.

15. Sjőberg, R.L.; Ducci, F.; Barr, C.S.; Newman, T.K.; Dell'Osso, L.; Virkkunen M, et al. Non-Additive Interaction of a Functional $M A O-A$ VNTR and Testosterone Predicts Antisocial Behavior. Neuropsychopharmacology 2008; 33(2): 425-430, doi:10.1038/sj.npp.1301417.

16. Caspi, A.; McClay, J.; Moffitt, T.Y.; Mill, J.; Martin, J.; Craig, I.W.; et al. Role of genotype in the cycle of violence in maltreated children. Science 2002; 297: 851-854.

17. Pintar, J.E.; Barbosa, J.; Francke, U.; Castiglione, C.M.; Hawkins, M.; Breakefield, X.O. Gene for monoamine oxidase type A assigned to the human $X$ chromosome. The Journal of Neuroscience 1981; 1(2): 166-175.

18. Cases, O.: Seif, I.; Grimsby, J.; Gaspar, P.; Chen, K.; Pournin, S.; et al. Aggressive Behavior and Altered Amounts of Brain Serotonin and Norepinephrine in Mice Lacking MAOA. Science 1995; 268(5218): 1763-1766.

19. McDermott, R.; Tingley, D.; Cowden, J.; Frazzetto, G.; Johnson, D.D.P. Monoamine oxidase A gene (MAOA) predicts behavioral aggression following provocation. Proc Nat. Acad. Sci. 2009; 106(7): 21182123, doi:10.1073/pnas.0808378106.

20. Ziermans, T.; Dumontheil, I.; Roggeman, C.; Peyrard-Janvid, M.; Matsson, H.; Kere, J.; et al. Working memory brain activity and capacity link $M A O A$ polymorphism to aggressive behavior during development. Transl. Psychiatry 2012; 2 e85.

21. Marquez, C.; Poirier, G.L.; Cordero, M.I.; Larsen, M.H.; Groner, A.; Marquis, J.; et al. Peripuberty stress leads to abnormal aggression, altered amygdale and orbitofrontal reactivity and increased prefrontal MAOA gene expression. Transl. Psychiatry 2013; e216.

22. Hart, H.; Lim, L.; Mehta, M.A.; Chatzieffraimidou, A.; Curtis, C.; Xu, X.; et al. Reduced functional connectivity of fronto-pariental sustained attention networks in severe childhood abuse. PloS ONE 2017; 12(11): e0188744, 10.1371/journal.pone.01887444.

23. Meyer-Lindenberg, A.; Buckholtz, J.W.; Kolachana, B.; Hariri, A.R.; Pezawas, L.; Blasi, G.; et al. Neural mechanism of genetics risk for impulsivity and violence in humans. PNAS 2006; 103(16): 6269-6274, doi:10.1073/pnas.0511311103.

24. Zhang, Y.; Ming, Q-s.; Yi J-y, Wang, X.; Chai, Q-1.; Yao, S-q. Gene-Gene-Environment Interactions of Serotonin Transporter Monoamine Oxidase and Childhood Maltreatment Predict Aggressive Behavior in Chinese Adolescents. Front. Behav. Neurosci. 2017; 11: 17, doi:10.3389/fnbeh.2017.00017.

25. Guo, G.; OU, X-M.; Roettger, M.; Shih, J.C. The VNTR 2 repeat in MAOA and delinquent behavior in adolescence and young adulthood: associations and MAOA promoter activity. European Journal of Human Genetics 2008; 16: 626-634, doi:10.1038/sj.ejhg.5201999.

26. Young, S.E.; Smolen, A.; Hewitt, J.K.; Haberstick, B.C.; Stallings, M.C.; Corley, R.P.; Crowley, T.J. Interaction Between MAO-A Genotype and Maltreatment in the Risk for conduct Disorder: Failure to Confirm in Adolescent Patients. Am J Psychiatry 2006; 163: 1019-1025. 
27. Ouellet-Morin, I.; Côté, S.M.; Vitaro, F.; Hébert, M.; Carbonneau, R.; Lacourse, E.; et al. Effects of the MAOA gene and levels of exposure to violence on antisocial outcomes. The British Journal of Psychiatry 2016; 208: 42-48, doi:10.1192/bjp.bp.114.162081.

28. Godar, S.C.; Fite, P.J.; McFarlin, K.M.; Bortolato, M. The role of monoamine oxidase A in aggression: current translational developments and future challenges. Prog Neuropsychoparmachol Biol Psychiatry 2016; 69: 90-100. doi:10.1016/j.pnpbp.2016.01.001.

29. McSwiggan, S.; Elger, B.; Appelbaum, P.S. The Forensic Use of Behavioral Genetics in Criminal Proceedings: Case of the MAOA-L Genotype. Int J Law Psychiatry 2017; 50: 17-23. doi:10.1016/j.ijlp.2016.09.005.

30. Piton, A.; Poquet, H.; Redin, C.; Masurel, A.; Laurel, J.; Muller, J.; et al. 20 ans après: a second mutation in MAOA identified by targeted high-throughput sequencing in a family with altered behavior and cognition. European Journal of Human Genetics 2013; 22: 776-783. doi:10.1038/ejhg.2013.243.

31. Ding, Y.; Li, H.; Chen, L-L.; Xie, K. Recent Advances in Genome Editing Using CRISPR//Cas9. Frontiers in Plant Science 2016; Volume 7, Article 703, doi:10.3389/fpls.2016.00703.

32. Eid, A.; Mahfouz, M.M. Genome editing: the road of CRISPR/Cas9 from bench to clinic. Experimental and Molecular Medicine 2016; 46, e265, doi:10.1038/emm.2016.111.

33. Chu, V.T.; Weber, T.; Graf, R.; Sommermann, T.; Petsch, K.; Sack, U.; Volchkov, P.; Rajewsky, K.; Kühn, R. Efficient generation of Rosa26 knock-in mice using CRISPR/Cas9 in C57BL/6 zygotes. BMC Biotechnology 2016; 16:4, doi:10.1186/s12896-016-0234-4.

34. Kawahara, A.; Hisano, Y.; Ota, S.; Taimatsu, K Site-Specific Integration Exogenous Genes Using Genome Editing Technologies in Zebrafish. Int. J. Mol. Sci. 2016; 17: 727. doi:10.3390/ijms17050727.

35. Yao, X.; Wang, X.; Hu, X.; Liu, Z.; Liu, J.; Zhou, H.; et al. Homology-mediated end joining-based targeted integration using CRISPR/Cas9. Cell Research 2017; 27: 801-814, doi:10.1038/cr.2017.76.

36. Mao, Z.; Bozzella, M.; Seluanov, A.; Gorbuanova, V. DNA repair by nonhomologous end joining and homologous recombination during cell cycle in human cells. Cell Cycle 2009; 7(18): 2902-2906.

37. Nakade, S.; Tsubota, T.; Sakane, Y.; Kume, S.; Sakamoto, M.; Obara, M. Microhomology-mediated end-joining-dependent integration of donor DNA in cells and animals using TALENs and CRISPR/Cas9. Nature Communication 2014: 5: 5560, 10:1038/ncomms6560.

38. Park, C.Y.; Kim, D.H.; Son, J.S.; Sung, J.J.; Lee, J.; Bae, S.; et al. Functional Correction of Large Factor VIII Gene Chromosomal Inversions in Hemophilia A Patient-Derived iPSCs Using CRISPR/Cas9. Cell Stem Cell 2015; 17(2): 213-220.

39. Guan, Y.; Ma, Y.; Li, Q.; Sun, Z.; Ma, L.; Wu, L.; et al. CRISPR/Cas9-mediated somatic correction of a novel coagulation factor IX gene mutation ameliorates hemophilia in mouse. EMBO Molecular Medicine 2016; 8: 477-488, doi:10.15252/emmm.201506039. 